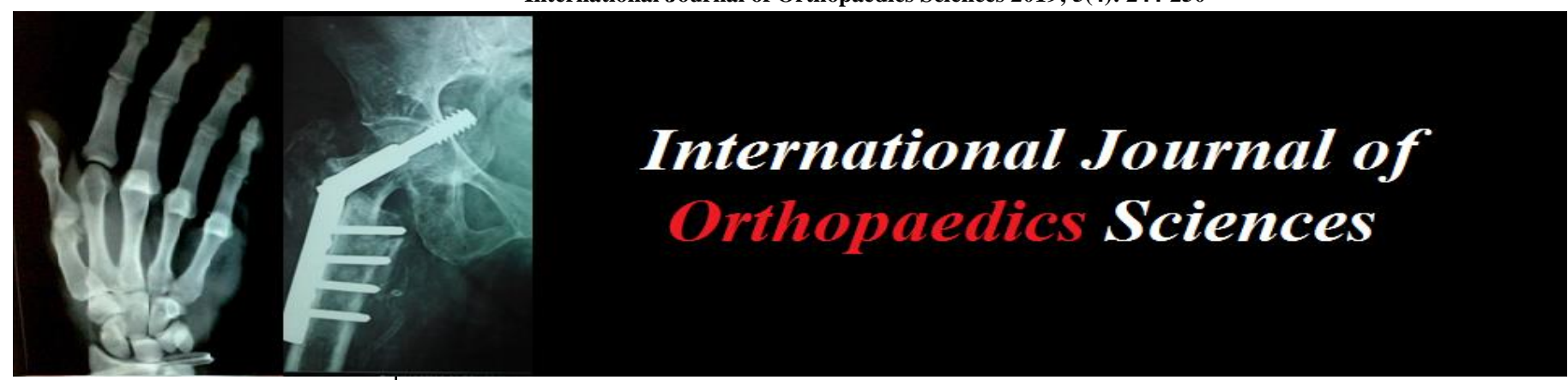

E-ISSN: 2395-1958

P-ISSN: 2706-6630

IJOS 2019; 5(4): 244-250

(C) 2019 IJOS

www.orthopaper.com

Received: 04-08-2019

Accepted: 08-09-2019

Sushanta Kumar Das

Associate Professor

Coordinator, Department of

Pharm. D, (Attached with

Gandhi Medical College \&

Hospital), CMR College of

Pharmacy, Medchal, Hyderabad,

Telangana, India

Dr. J Satyanarayana

Professor and Head, Department of Orthopedics, Gandhi Medical

College \& Hospital,

Secunderabad, Telangana, India

Corresponding Author:

Dr. J. Satyanarayana

Professor and Head, Department

of Orthopedics, Gandhi Medical

College \& Hospital,

Secunderabad, Telangana, India

\section{A prospective observational study on Antibiotic prescription and their switch pattern in various orthopedic wounds in a tertiary care teaching hospital}

\section{Sushanta Kumar Das and Dr. J Satyanarayana}

DOI: https://doi.org/10.22271/ortho.2019.v5.i4e.1677

Abstract

Introduction: Orthopedic wounds are traumatic wounds due to hard and/or soft tissue trauma. Antibiotics are most commonly and frequently prescribed drugs for long periods in orthopedic department. Use of antibiotics depends on clinical need of patient, known sensitivities to infecting organism and also depends on the evidence gathered by the orthopedic surgeons throughout their clinical practice. Hence our study aimed to identify various antibiotics prescribed in the orthopedic wound and also to highlight the switch patterns of these antibiotics.

Methodology: A prospective observational study was conducted for a period of eight months; data was collected and analyzed according to inclusion and exclusion criteria and ANOVA was applied to obtain the statistical outcome of results.

Discussion: Out of total 115 cases, Closed fractures (44.34\%) and Open fractures $(41.74 \%)$ had high incidence. Total 17 antibiotics were prescribed for 365 times, with Cefotaxime (25.50\%), Cefuroxime $(17.8 \%)$ and Amikacin (14.52\%) were most frequent. 9 Different types of drug switches (1-1, 1-2, 1-3, 2$1,2-2,2-3,3-1,3-2,3-3)$ were present in the study which are mainly due to antibiotic resistance \& sensitivity, wound status (size, severity, type) and type of micro organism to obtain better therapeutic outcome.

Conclusion: Our study concludes that, frequency of antibiotic(s) prescription is quite high in the department. Furthermore lack of serology assay makes it difficult to choose appropriate antibiotic and leads to increased number of antibiotic switch. This study highlights 5, $6 \&$ even 7 antibiotics per case also. A systemic approach must be initiated to streamline the appropriate antibiotic prescription and a standard antibiotic guideline for the department must be formulated.

Keywords: Orthopedics, wound healing, antibiotics, switch

\section{Introduction}

A wound can be described in various ways; by etiology, anatomical location, acute or chronic type, method of closure, along with presenting symptoms or definitely by the form of the predominant tissue types in the wound bed. All descriptions are necessary with a critical purpose for the assessment and proper management of the wound with symptom resolution and healing. A wound by true definition is a breakdown in the protective function of the skin; the loss of continuity of epithelium, with or without loss of underlying connective tissue (i.e. muscle, bone, nerves) following injury to the skin or underlying tissues/organs caused by surgery, a blow, a cut, chemicals, heat/cold, friction/shear force, pressure or as a result of disease, such as leg ulcers or carcinomas ${ }^{[1]}$. Wound healing is body's response to injury; in an attempt to restore normal structure and functions which involves mainly two processes: regeneration and repair ${ }^{[2]}$.

Orthopedic wounds are a form of traumatic wounds with or without need of surgical intervention and occurs in response to hard and/or soft tissue trauma which includes; bone, muscle, ligament and tendons trauma. The most common orthopedic wounds include:

- Infection with discharge (Osteomyelitis \& post operative osteomyelitis).

- Fractures (Open \& closed).

- Surgical (Implants, prosthetics, amputation and non implant surgery like deformity correction). 
Antibiotics are most often and frequently prescribed medicine for longer duration in orthopedic department, antibiotics are prescribed both as prophylactic (before orthopedic surgical intervention) or to manage present infection ${ }^{[3]}$. Prophylactic antibiotics can reduce the risk of wound infection and have been regularly prescribed in orthopedic surgery for decades. Despite their extensive application, selection of antibiotics and their timing, duration of administration and switch to other group remain an unsolved question. The health economic costs associated with orthopedic wound infections are significant but sensible and suitable use of antibiotics can reduce this many fold ${ }^{[4]}$. Initial choice and switch of antibiotics depends on clinical need of patient as well as on known sensitivities of the infecting organism ${ }^{[3]}$. For a while the selection of antibiotic also depends on the experience and evidence gathered by the orthopedic surgeons throughout their clinical practice. Hence we want to identify various antibiotics prescribed in the orthopedic wound condition and also to highlight the switch patterns of these antibiotics in the department.

\section{Methodology}

This prospective case analysis study was conducted for a period of 8 months between August 2017 and March 2018 in Department of Orthopedics, Gandhi Hospital, Secunderabad with prior Approval from Institutional Ethical Committee, CMR College of Pharmacy and with necessary permission from Department of Orthopedics, Gandhi Hospital, Secunderabad. Selected Cases were collected and documented in a structured data compilation form from the in-patient department of orthopedics on a daily basis according to inclusion criteria, which includes; patient of all ages and both genders, cases diagnosed with various orthopedic clinical condition in which wound is positive \& with definite antibiotic(s) prescription, patients for whom orthopedic implant, prosthetic, amputation or deformity corrections were planned or performed, Cases with complete information till discharge. Study exclusion criteria includes; patient diagnosed with non-wound orthopedic diseases and/or clinical condition like, rheumatoid arthritis, osteoarthritis, anterior cruciate ligament tear etc., cases with incomplete information or without a proper discharge summary, if patient do not want to participate in the study after describing the study process, HIV positive cases and if patient absconded or expired. A total of 115 cases with justified inclusion criteria were collected during the study period which was reviewed on a regular basis to update and further follow-up till discharge. Outcome was framed after interpreting the data gathered from case documentation forms; according to various category and parameters. Further results were discussed thoroughly with orthopedic surgeons in a regular manner to accomplish the outcome.

\section{Statistics}

The interpreted data were statistically analyzed by using 'Graph Pad Instat' software. ANOVA, Tukey-Kramer multiple comparison test and one sample $\mathrm{T}$ test were performed to analyze various parameters to obtain the statistical significance of each parameter ${ }^{[5]}$.

\section{Results}

Table 1 shows the demographic details of the collected cases.
Out of 115 cases, it indicates male predominance (87\%) in this study. Maximum number of cases falls within the age group of $21-40$ years $(53.92 \%)$ followed by $41-60$ years $(27.82 \%)$ \& up to 20 years $(13.92 \%)$. Disease wise distribution indicates that maximum number of cases with fractures, both open (44\%) and closed fracture (42\%) have almost equal occurrence. Other conditions have very minor presence in the study. Figure 1, Figure 2 and Figure 3.

Table 2 indicates that Cefotaxime $(25.50 \%)$ is the drug of choice in the orthopedic department followed by Cefuroxime (17.8\%), Amikacin (14.52\%), Metronidazole (11.8\%), Amoxicillin-Clavulanic Acid (10.42\%), PipercillinTazobactum $(8.5 \%)$, whereas Ceftriaxone and Cefixime were prescribed in limited cases and Gentamycin, Vancomycin, Ciprofloxacin, Azithromycin, Streptomycin, Imepenem, Faropenem, Meropenem and Clarithromycin were prescribed in very few cases.

Table 3 reflects the number of antibiotic(s) per case. It shows almost equal number of cases (approximately 30) has either 2, 3 or 4 Antibiotics prescribed; surprisingly 6 antibiotics were also prescribed in 8 cases and 7 Antibiotics in one case.

Table 4 indicates the antibiotic switch pattern, majority of antibiotic route switch was found to be parenteral - oral $(85 \%)$. This table further indicates predominance in 1 switch of antibiotics $(28.58 \%)$ followed by 2 switches $(24.10 \%), 3$ switches $(20.53 \%), 4$ switches $(11.60 \%), 5$ switches $(6.26 \%)$, No switch $(6.26 \%), 7$ switches $(1.78 \%)$ and 6 switches $(0.89 \%)$.

Table 5.1 shows a Cefuroxime to Cefotaxime (19.6\%) switch as the maximum number of 1-1 type of drug switch in antibiotics followed by Cefotaxime to Cefuroxime (12.88\%), Cefotaxime to Amoxicillin-Clavulante (6.07\%).

Table 5.2 indicates drug switch from Cefotaxime to Cefuroxime \& Amikacin $(25 \%)$ are the maximum, followed by Cefotaxime to Cefotaxime \& Amikacin (12.50\%), Ciprofloxacin to Ciprofloxacin \&Metronidazole (12.50\%) in 1-2 type of antibiotic drug switch.

Table 5.3 indicates the drug switch from PiperacillinTazobactam to Cefuroxime, Amikacin \& Metroindazole is seen in two cases and the other switches are seen in only one case each in 1-3 type of antibiotic drug switches.

Table 5.4 indicates drug switch from cefuroxime \& Amikacin to Cefuroxime $(10.42 \%)$ as the maximum in $2-1$ type of antibiotic drug switch.

Table 5.5 indicates drug switch from Ceftriaxone \& Amikacin to Cefixime \& Amoxicillin-Clavulanate is seen in two cases and the other switches are seen in only one case each in 2-2 type of switch.

Table 5.6 indicates drug switch from Cefotaxime \& Metronidazole to Piperacillin - Tazobactam, Amikacin \& Metronidazole is seen in two cases and the other switches are seen in only one case each in 2-3 type of switch.

Table 5.7 indicates drug switch from Cefotaxime, Metronidazole \& Amikacin to Cefotaxime (30\%) as the maximum in 3-1 type of antibiotic drug switch.

Table 5.8 indicates drug switch from Cefotaxime, Metronidazole \& Amikacin to Cefotaxime \& Metronidazole is seen in five cases and the other switches are seen in only one case each in 3-2 type of switch.

Table 5.9 indicates that all the drug switches are with equal percentages (25\%) in 3-3 type of antibiotic switch. 
Table 1: Demographic details of collected cases $(n=115)$

\begin{tabular}{|c|c|c|c|}
\hline \multicolumn{3}{|c|}{ Gender categorization: } \\
\hline S. No & Gender & No. of Patients & Percentage (\%) \\
\hline 1 & Male & 100 & 87.0 \\
\hline 2 & Female & 15 & 13.0 \\
\hline
\end{tabular}

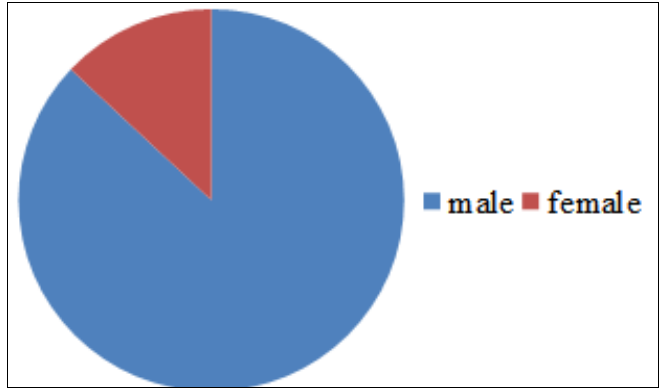

Fig 1: Demographic details of collected cases

Age wise distribution

\begin{tabular}{|c|c|c|c|}
\hline S. No & Age (Yrs) & No. of Patients & Percentage (\%) \\
\hline 1 & Up to 20 & 16 & 13.92 \\
\hline 2 & $21-40$ & 62 & 53.92 \\
\hline 3 & $41-60$ & 32 & 27.82 \\
\hline 4 & $\geq 60$ & 05 & 4.34 \\
\hline
\end{tabular}

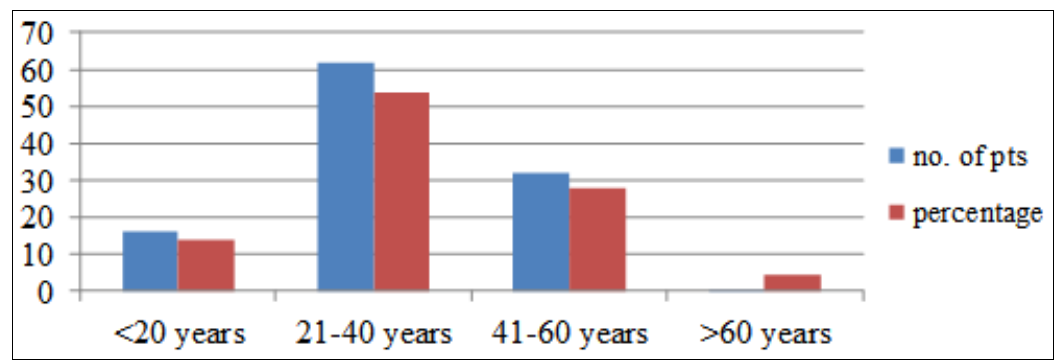

Fig 2: Age wise distribution

Distribution of cases based on various orthopedic wounds

\begin{tabular}{|c|c|c|c|}
\hline S. No & Disease/Clinical condition & No. of cases & Percentage (\%) \\
\hline 1 & Closed fractures & 51 & 44.34 \\
\hline 2 & Open fractures & 48 & 41.74 \\
\hline 3 & Chronic osteomyelitis (OM) & 06 & 5.22 \\
\hline 4 & Implants & 04 & 3.48 \\
\hline 5 & Amputations & 04 & 3.48 \\
\hline 6 & Acute osteomyelitis & 02 & 1.74 \\
\hline
\end{tabular}

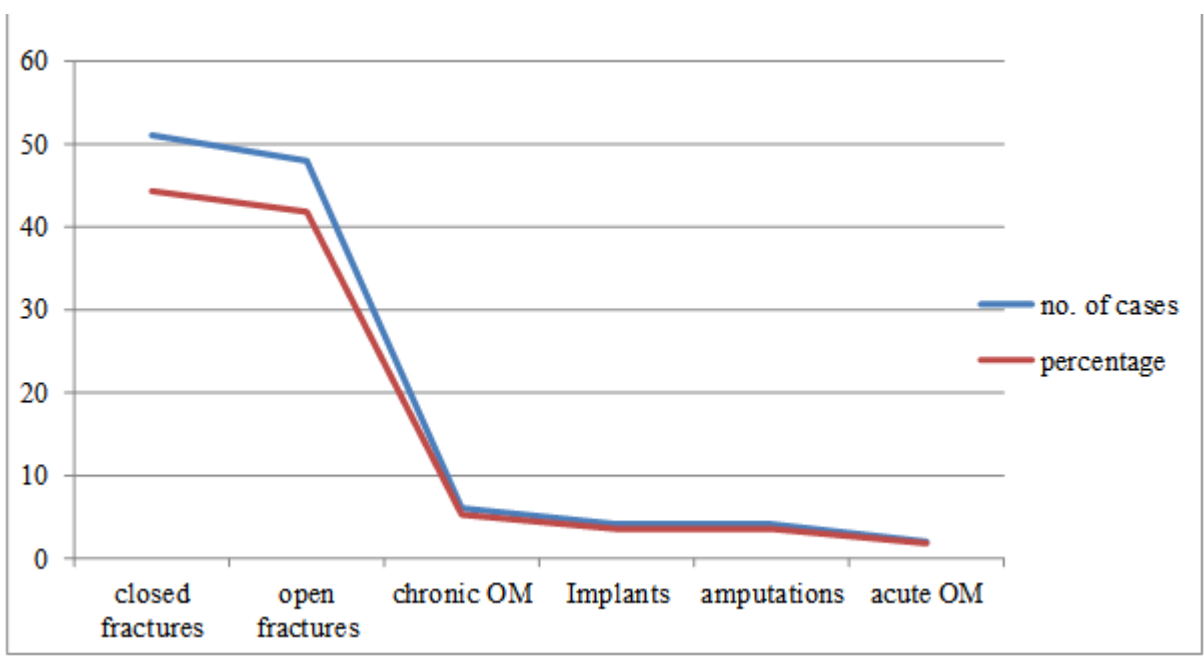

Fig 3: Distribution of cases based on various orthopedic wounds 
Table 2: Distribution of total Antibiotics frequency prescribed in collected cases $(n=365)$

\begin{tabular}{|c|c|c|c|}
\hline S. No & Name of Antibiotic & Frequency & Percentage (\%) \\
\hline 1 & Cefotaxime & 93 & 25.50 \\
\hline 2 & Cefuroxime & 65 & 17.80 \\
\hline 3 & Amikacin & 53 & 14.52 \\
\hline 4 & Metronidazole & 43 & 11.80 \\
\hline 5 & Amoxicillin-Clavulanic Acid & 38 & 10.42 \\
\hline 6 & Pipercillin-Tazobactum & 31 & 8.50 \\
\hline 7 & Ceftriaxone & 18 & 4.93 \\
\hline 8 & Cefixime & 10 & 2.73 \\
\hline 9 & Gentamycin & 03 & 0.82 \\
\hline 10 & Ciprofloxacin & 03 & 0.82 \\
\hline 11 & Vancomycin & 02 & 0.54 \\
\hline 12 & Azithromycin & 01 & 0.27 \\
\hline 13 & Streptomycin & 01 & 0.27 \\
\hline 14 & Imepenam & 01 & 0.27 \\
\hline 15 & Faropenem & 01 & 0.27 \\
\hline 16 & Meropenem & 01 & 0.27 \\
\hline 17 & Clarithromycin & 01 & 0.27 \\
\hline
\end{tabular}

Table 3: Number of Antibiotics prescribed per case $(n=115)$

\begin{tabular}{|c|c|c|c|}
\hline S. No & No. of Antibiotic prescribed/Case & No. of cases & Percentage (\%) \\
\hline 1 & Only 1 Antibiotic & 10 & 8.70 \\
\hline 2 & 2 Antibiotics & 28 & 24.34 \\
\hline 3 & 3 Antibiotics & 31 & 27.20 \\
\hline 4 & 4 Antibiotics & 32 & 27.80 \\
\hline 5 & 5 Antibiotics & 05 & 4.34 \\
\hline 6 & 6 Antibiotics & 08 & 6.95 \\
\hline 7 & 7 Antibiotics & 01 & 0.67 \\
\hline
\end{tabular}

Mean - 3.278, SD - 1.367

SEM - 0.1275, P value - $<0.005$

Table 4: Antibiotic switch distribution

\begin{tabular}{|c|c|c|c|}
\hline \multicolumn{2}{|c|}{ Distribution based on frequency of parenteral - oral and oral - parenteral switch of antibiotics (n=164) } \\
\hline S. No & Type of switch & Frequency & Percentage (\%) \\
\hline 1 & Parenteral-Oral & 140 & 85.37 \\
\hline 2 & Oral-Parenteral & 24 & 14.63 \\
\hline \multicolumn{4}{|c|}{ Distribution based on drug-drug switch of antibiotics in collected cases (n=115) } \\
\hline S. No & Type of switch & No. of cases & Percentage (\%) \\
\hline 1 & No Switch & 10 & 8.70 \\
\hline 2 & 1 Switch & 32 & 27.83 \\
\hline 3 & 2 Switches & 27 & 20.48 \\
\hline 4 & 3 Switches & 23 & 11.31 \\
\hline 5 & 4 Switches & 13 & 6.08 \\
\hline 6 & 5 Switches & 07 & 0.86 \\
\hline 7 & 6 Switches & 01 & 1.74 \\
\hline 8 & 7 Switches & 02 & Mean - 2.417, SD - 1.475 \\
\hline & & \multicolumn{2}{c|}{ SEM - 0.1375, p value - < 0.05} \\
\hline
\end{tabular}

Table 5: Detail description of different types of Antibiotic switch

Table 5.1: 1 drug -1 drug type of antibiotic switch $(n=132)$

\begin{tabular}{|c|c|c|c|c|}
\hline S. No & Drug 1 & Drug 1 switch & No. of switches & Percentage (\%) \\
\hline 1 & Cefuroxime & Cefotaxime & 26 & 19.70 \\
\hline 2 & Cefotaxime & Cefuroxime & 17 & 12.88 \\
\hline 3 & Cefotaxime & Amoxicillin-Clavulante & 08 & 6.07 \\
\hline 4 & Piperacillin-Tazobactam & Cefotaxime & 07 & 5.30 \\
\hline 5 & Cefuroxime & Amoxicillin-Clavulante & 07 & 5.30 \\
\hline 6 & Cefotaxime & Cefixime & 07 & 5.30 \\
\hline 7 & Ceftriaxone & Cefotaxime & 04 & 3.03 \\
\hline 8 & Piperacillin-Tazobactam & Amoxicillin-Clavulante & 04 & 3.03 \\
\hline 9 & Cefotaxime & Ceftriaxone & 04 & 3.03 \\
\hline 10 & Cefixime & Amoxicillin-Clavulante & 03 & 2.27 \\
\hline 11 & Piperacillin-Tazobactam & Ceftriaxone & 03 & 2.27 \\
\hline 12 & Metroindazole & Amoxicillin-Clavulante & 03 & 2.27 \\
\hline 13 & Amoxicillin-Clavulante & Cefotaxime & 02 & 1.51 \\
\hline
\end{tabular}




\begin{tabular}{|l|c|c|c|c|}
\hline 14 & Vancomycin & Cefotaxime & 02 & 1.51 \\
\hline 15 & Cefuroxime & Piperacillin-Tazobactam & 02 & 1.51 \\
\hline 16 & Cefotaxime & Amikacin & 02 & 1.51 \\
\hline 17 & Amikacin & Amoxicillin-Clavulante & 02 & 1.51 \\
\hline 18 & Cefotaxime & Piperacillin-Tazobactam & 02 & 1.51 \\
\hline 19 & Cefuroxime & Cefixime & 02 & 1.51 \\
\hline 20 & Cefotaxime & Amoxicillin-Clavulante & 02 & 1.51 \\
\hline 21 & Cefuroxime & Metroindazole & 02 & 1.51 \\
\hline 22 & Cefixime & Cefuroxime & 01 & 0.76 \\
\hline 23 & Cefuroxime & Amoxicillin-Clavulante & 01 & 0.76 \\
\hline 24 & Metroindazole & Faropenem & 01 & 0.76 \\
\hline 25 & Cefuroxime & Amoxicillin-Clavulante & 01 & 0.76 \\
\hline 26 & Metroindazole & Cefuroxime & 01 & 0.76 \\
\hline 27 & Cefuroxime & Vancomycin & 01 & 0.76 \\
\hline 28 & Piperacillin-Tazobactam & Cefuroxime & 01 & 0.76 \\
\hline 29 & Ceftriaxone & Amikacin & 01 & 0.76 \\
\hline 30 & Cefuroxime & Amikacin & 01 & 0.76 \\
\hline 31 & Cefixime & Ceftriaxone & 01 & 0.76 \\
\hline 33 & Ceftriaxone & Cefixime & 01 & 0.76 \\
\hline 34 & Azithromycin & Cefotaxime & 01 & 0.76 \\
\hline 35 & Piperacillin-Tazobactam & Cefixime & 01 & 0.76 \\
\hline 36 & Piperacillin-Tazobactam & Amikacin & 01 & 0.76 \\
\hline 37 & Metroindazole & Piperacillin-Tazobactam & 01 & 0.76 \\
\hline 38 & Ceftriaxone & Amoxicillin-Clavulante & 01 & 0.76 \\
\hline 39 & Metroindazole & Piperacillin-Tazobactam & 01 & 0.76 \\
\hline 40 & Amoxicillin-Clavulante & Cefixime & 01 & 0.76 \\
\hline 41 & Cefixime & Cefotaxime & 01 & 0.76 \\
\hline 42 & Ceftriaxone & Ciprofloxacin & 01 & 0.76 \\
\hline 43 & Cefuroxime & Gentamycin & 01 & 0.76 \\
\hline
\end{tabular}

Table 5.2: 1 drug -2 drug type of antibiotic switch $(n=16)$

\begin{tabular}{|c|c|c|c|c|}
\hline S. No & Drug 1 & Drug 1 + Drug 2 switch & No. of Switches & Percentage (\%) \\
\hline 1 & Cefotaxime & Cefuroxime + Amikacin & 03 & 18.75 \\
\hline 2 & Cefotaxime & Cefotaxime + Amikacin & 02 & 12.50 \\
\hline 3 & Ciprofloxacin & Ciprofloxacin + Metroindazole & 02 & 12.50 \\
\hline 4 & Faropenem & Cefuroxime + Amikacin & 01 & 6.25 \\
\hline 5 & Imipenem & Cefotaxime + Piperacillin-Tazobactam & 01 & 6.25 \\
\hline 6 & Ceftriaxone & Piperacillin-Tazobactam + Metroindazole & 01 & 6.25 \\
\hline 7 & Piperacillin-Tazobactam & Cefotaxime + Amikacin & 01 & 6.25 \\
\hline 8 & Ceftriaxone & Ceftriaxone + Amikacin & 01 & 6.25 \\
\hline 9 & Ceftriaxone & Cefotaxime + Amikacin & 01 & 6.25 \\
\hline 10 & Cefotaxime & Amikacin + Cefuroxime & 01 & 6.25 \\
\hline 11 & Cefixime & Cefotaxime + Amikacin & 01 & 6.25 \\
\hline 12 & Cefotaxime & Cefuroxime + Metroindazole & 01 & 6.25 \\
\hline
\end{tabular}

Table 5.3: 1 drug -3 drug type of antibiotic switch $(n=4)$

\begin{tabular}{|c|c|c|c|c|}
\hline S. No & Drug 1 & Drug 1 + Drug 2 + Drug 3 switch & No. of Switches & Percentage (\%) \\
\hline 1 & Piperacillin- Tazobactam & Cefuroxime + Amikacin + Metroindazole & 02 & 50.0 \\
\hline 2 & Ceftriaxone & Cefotaxime + Amikacin + Cefixime & 01 & 25.0 \\
\hline 3 & Cefuroxime & Cefotaxime + Gentamycin + Metroindazole & 01 & 25.0 \\
\hline
\end{tabular}

Table 5.4: 2 drugs -1 drug type of antibiotic switch $(n=49)$

\begin{tabular}{|c|c|c|c|c|}
\hline S. No & Drug 1 + Drug 2 & Drug 3 & No. of Switches & Percentage (\%) \\
\hline 1 & Cefuroxime + Amikacin & Cefuroxime & 05 & 10.42 \\
\hline 2 & Cefotaxime + Metronidazole & Cefotaxime & 05 & 10.42 \\
\hline 3 & Cefuroxime + Amikacin & Cefotaxime & 04 & 8.39 \\
\hline 4 & Cefotaxime + Piperacillin-Tazobactam & Cefotaxime & 03 & 6.25 \\
\hline 5 & Cefotaxime + Amikacin & Cefotaxime & 03 & 6.25 \\
\hline 6 & Cefuroxime + Cefotaxim & Cefotaxime & 02 & 4.20 \\
\hline 7 & Cefuroxime + Amikacin & Amoxicillin-Clavulanate & 02 & 4.20 \\
\hline 8 & Cefotaxime + Amikacin & Cefixime & 02 & 4.20 \\
\hline 9 & Ciprofloxacin + Ceftriaxone & Piperacillin-Tazobactam & 01 & 2.08 \\
\hline 10 & Piperacillin-Tazobactam + Metronidazole & Cefotaxime & 01 & 2.08 \\
\hline 11 & Cefotaxime + Amikacin & Cefixime & 01 & 2.08 \\
\hline 12 & Piperacillin-Tazobactam + Metronidazole & Cefotaxime & 01 & 2.08 \\
\hline 13 & Cefotaxime + Amikacin & Metronidazole & 01 & 2.08 \\
\hline 14 & Amikacin + Metronidazole & Metronidazole & 01 & 2.08 \\
\hline
\end{tabular}




\begin{tabular}{|l|c|c|c|c|}
\hline 15 & Cefuroxime + Metronidazole & Cefuroxime & 01 & 2.08 \\
\hline 16 & Amoxicillin-Clavulanate + Metronidazole & Cefotaxime & 01 & 2.08 \\
\hline 17 & Amoxicillin-Clavulanate + Cefotaxime & Amoxicillin-Clavulanate & 01 & 2.08 \\
\hline 18 & Piperacillin-Tazobactam + Metronidazole & Cefuroxime & 01 & 2.08 \\
\hline 19 & Piperacillin-Tazobactam + Amikacin & Cefotaxime & 01 & 2.08 \\
\hline 20 & Ciprofloxacin + Metronidazole & Ciprofloxacin & 01 & 2.08 \\
\hline 21 & Ciprofloxacin + Metronidazole & Cefuroxime & 01 & 2.08 \\
\hline 22 & Ceftriaxone + Metronidazole & Ceftriaxone & 01 & 2.08 \\
\hline 23 & Cefotaxime + Amikacin & Amoxicillin-Clavulanate & 01 & 2.08 \\
\hline 24 & Ceftriaxone + Amikacin & Ceftriaxone & 01 & 2.08 \\
\hline 25 & Cefixime + Metronidazole & Cefixime & 01 & 2.08 \\
\hline 26 & Gentamycin + Vancomycin & Azithromycin & 01 & 2.08 \\
\hline 27 & Ciprofloxacin + Cefotaxime & Cefotaxime & 01 & 2.08 \\
\hline 28 & Ceftriaxone + Amikacin & Amoxicillin-Clavulanate & 01 & 2.08 \\
\hline 29 & Ceftriaxone + Metronidazole & Cefixime & 01 & 2.08 \\
\hline 30 & Cefuroxime + Metronidazole & Piperacillin-Tazobatam & 01 & 2.08 \\
\hline 31 & Cefuroxime + Metronidazole & Cefotaxime & 01 & 2.08 \\
\hline
\end{tabular}

Table 5.5: Table 13: 2-2 Type of antibiotic switch $(n=10)$

\begin{tabular}{|c|c|c|c|c|}
\hline S. No & Drug 1 + Drug 2 & Drug 1 + Drug 2 & No of Switches & Percentage (\%) \\
\hline 1 & Ceftriaxone+ Amikacin & Cefixime + Amoxicillin-Clavulanate & 02 & 20.0 \\
\hline 2 & Cefotaxime + Amikacin & Metronidazole + Amoxicillin-Clavulante & 01 & 10.0 \\
\hline 3 & Ceftriaxone + Metronidazole & Cefixime + Metronidazole & 01 & 10.0 \\
\hline 4 & Ceftriaxone + Metronidazole & Gentamycin + Vancomycin & 01 & 10.0 \\
\hline 5 & Cefotaxime + Amikacin & Cefotaxime + Amoxicillin-Clavulanate & 01 & 10.0 \\
\hline 6 & Cefotaxime + Amikacin & Cefotaxime + Metronidazole & 01 & 10.0 \\
\hline 7 & Metronidazole + Cefotaxime & Piperacillin-Tazobactam + Streptomycin & 01 & 10.0 \\
\hline 8 & Ceftriaxone + Amikacin & Cefuroxime + Amikacin & 01 & 10.0 \\
\hline 9 & Piperacillin-Tazobactam + Metronidazole & Cefotaxime + Piperacillin-Tazobactam & 01 & 10.0 \\
\hline
\end{tabular}

Table 5.6: 2 drugs -3 drugs type of antibiotic switch $(n=5)$

\begin{tabular}{|c|c|c|c|c|}
\hline S. No & Drug 1 + Drug 2 & Drug 1 + Drug 2 + Drug 3 & No of Switches & Percentage (\%) \\
\hline 1 & Cefotaxime + Metronidazole & Piperacillin-Tazobactam + Amikacin + Metronidazole & 02 \\
\hline 2 & Cefotaxime + Metronidazole & Cefuroxime + Amikacin + Metronidazole & 40.0 \\
\hline 3 & Cefotaxime + Amikacin & Ceftriaxone + Metronidazole + Ciprofloxacin & 01 & 01 \\
\hline 4 & Piperacillin-Tazobactam + Streptomycin & Cefotaxime + Streptomycin+ Imepenem & 20.0 & 20.0 \\
\hline
\end{tabular}

Table 5.7: 3 drugs -1 drug type of antibiotic switch $(n=20)$

\begin{tabular}{|c|c|c|c|c|}
\hline S. No & Drug $1+$ Drug $2+$ Drug 3 & Drug 1 & No of Switches & Percentage $(\%)$ \\
\hline 1 & Cefotaxime + Metronidazole + Amikacin & Cefotaxime & 06 & 30.0 \\
\hline 2 & Cefuroxime + Amikacin + Metronidazole & Cefuroxime & 05 & 25.0 \\
\hline 3 & Cefuroxime + Amikacin + Metronidazole & Cefotaxime & 01 & 5.0 \\
\hline 4 & Amikacin + Metronidazole + Piperacillin - Tazobactam & Cefotaxime & 01 & 5.0 \\
\hline 5 & Cefotaxime + Streptomycin + Imepenem & Imepenem & 01 & 5.0 \\
\hline 6 & Amikacin + Metronidazole + Piperacillin - Tazobactam & Piperacillin- Tazobactam & 01 & 5.0 \\
\hline 7 & Amikacin + Metronidazole + Piperacillin - Tazobactam & Metronidazole & 01 & 5.0 \\
\hline 8 & Cefuroxime + Gentamycin + Metronidazole & Cefotaxime & 01 & 5.0 \\
\hline 9 & Piperacillin-Tazobactam + Gentamycin + Metronidazole & Amoxicillin- Clavulante & 01 & 5.0 \\
\hline 10 & Cefotaxime + Piperacillin-Tazobactam + amikacin & Piperacillin-Tazobactam & 01 & 5.0 \\
\hline 11 & Cefotaxime + Piperacillin-Tazobactam + Metronidazole & Cefotaxime & 01 & 5.0 \\
\hline
\end{tabular}

Table 5.8: 3 drugs -2 drugs type of antibiotic switch $(n=10)$

\begin{tabular}{|c|c|c|c|c|}
\hline S. No & Drug $1+$ Drug $2+$ Drug 3 & Drug 1 + Drug 2 & No of Switches & Percentage (\%) \\
\hline 1 & Cefotaxime + Metronidazole + Amikacin & Cefotaxime + Metronidazole & 05 & 50.0 \\
\hline 2 & Cefotaxime + Metronidazole + Amikacin & Amikacin + Metronidazole & 01 & 10.0 \\
\hline 3 & Cefuroxime + Amikacin + Metronidazole & Metronidazole + Piperacillin-Tazobactam & 01 & 10.0 \\
\hline 4 & Piperacillin-Tazobactam + Amikacin + Metronidazole & Amikacin + Metronidazole & 01 & 10.0 \\
\hline 5 & Piperacillin-Tazobactam + Amikacin + Metronidazole & Amikacin + Piperacillin- Tazobactam & 01 & 10.0 \\
\hline 6 & Cefuroxime + Amikacin + Metronidazole & Cefuroxime + Metronidazole & 01 & 10.0 \\
\hline
\end{tabular}

Table 5.9: 3 drugs -3 drugs type of antibiotic switch $(n=4)$

\begin{tabular}{|c|c|c|c|c|}
\hline S. No & Drug $1+$ Drug $2+$ Drug 3 & Drug $1+$ Drug $2+$ Drug 3 & No of Switches & Percentage $(\%)$ \\
\hline 1 & Cefuroxime + Amikacin + Metronidazole & Piperacillin-Tazobactam + Amikacin + Metronidazole & \begin{tabular}{|l|l}
01 &
\end{tabular} & 25.0 \\
\hline 2 & Cefuroxime + Amikacin + Metronidazole & Cefotaxime + Amikacin + Metronidazole & 01 & 25.0 \\
\hline 3 & Cefotaxime + Amikacin + Metronidazole & Cefuroxime + Amikacin + Metronidazole & 01 & 25.0 \\
\hline 4 & Piperacillin-Tazobactam+ Gentamycin + Amikacin & Piperacillin-Tazobactam + Gentamycin + Metronidazole & 01 & 25.0 \\
\hline
\end{tabular}




\section{Discussions}

A total of 115 cases were identified, included and analyzed for the study from In-patient units of Department of Orthopedics, Gandhi Hospital, Secunderabad. From the study we observed that a total of 17 different types of Antibiotic were prescribed throughout the collected cases with total frequency of 365. Amongst them 3 antibiotics were prescribed repeatedly i.e., Cefotaxime, Cefuroxime and Amikacin which contradicts the result of previous study where Ceftriaxone was most prescribed antibiotic (62\%) conducted by Das SK (2016) ${ }^{[6]}$ in the same department. In our study, frequency of antibiotic prescription was found to be 365 , which is quite a high number in terms of antibiotic, as antibiotic prescription should be precise and definite rather than trial \& error method which was also reported by Leekha S (2011) ${ }^{[7]}$ suggesting appropriate use, need and timing of Antimicrobials during the therapy. From our study it was found that male of 21-40 yrs age group are predominant and a total of 6 various orthopedic clinical conditions were documented, out of which; Fractures are higher in incidence. Both this findings were previously reported by Rajarathna $\mathrm{K}$ (2014) ${ }^{[8]}$. This may be the reason that male of this particular age group are frequently mobile with the use of personal transport (especially two-wheeler). From our study we found that number of Antibiotic prescribed per prescription are mostly 3 and 4, which was contradicted by Reji S (2015) [9] which reports Single Antibiotic use. From our study we observed that both oral and parenteral routes are preferred for administration of Antibiotic, similar finding was also reported by Ubedulla $S$ (2013) ${ }^{[10]}$ this is mainly due to initiation of antibiotics with IV route followed by switch to oral route once the effectiveness is established. From our study we observed that, parenteral to oral switch are predominant in Antibiotic administration, whereas oral to parenteral switch was. This is mainly due to severity of the condition and need of faster drug effect, once the condition gets stabilized or normalized, drugs switch to oral route. In this study we found that a total of 7 different drug switches per prescription, of which 1 drug switch per prescription was predominant this trend is also supported by - NHS policy [11]. 9 Different types of drug switches (1-1, 1-2, 1-3, 2-1, 2-2, 2-3, 3-1, 3-2, 3-3) are present in our study which are mainly done due to antibiotic resistance, sensitivity profile, wound status (Size, severity, type), culture sensitivity report, type of micro organism to obtain better therapeutic outcome as reported in Zhenjun $(2002)^{[12]}$.

\section{Conclusion}

Our study concludes that, frequency of antibiotic(s) prescription is quite high in the department. Furthermore lack of serology assay makes it difficult to choose appropriate antibiotic and leads to increased number of antibiotic switch. More than 3 antibiotics in a single case must be avoided to prevent antibiotic resistance but our study shows even 7 antibiotic per case also. A systemic approach must be initiated to streamline the appropriate antibiotic prescription and a standard antibiotic guideline for the department must be formulated with culture and sensitivity tests as a reference. Additionally clinical pharmacist should also play an important role to identify and report any prescription error to the concern doctor to improve patient outcome.

\section{References}

1. http://www.clinimed.co.uk/WoundCare/Education/Wound-Essentials/What-is-a-Wound- .aspx [Cited 2017 Oct 07]

2. Mohan H. Text book of Pathology. $6^{\text {th }}$ ed. New Delhi; Jaypee Brothers Medical Publishers (p) Ltd, 2010, 165p.

3. Radji M, Aini F, Fauziji S. Evaluation of Antibiotic prophylaxis, administration at the orthopedic surgery clinic of tertiary hospital in Jakarta, Indonesia. Asian Pac J Trop Dis. 2014; 4(3):190-3. Available from: https://www.ncbi.nlm.nih.gov/pmc/articles/PMC403203/ DOI:10.1016/S2222-1808(14)60503-

4. Bryson DJ, Morris DLJ, Shivji FS, Rollins KR, Snape $\mathrm{S}$, Ollivere BJ. Antibiotic prophylaxis in orthopedic surgery. Bone Joint J. 2016; 98-B(8):1014-9. Available from: https://www.ncbi.nlm.nih.gov/pubmed/27482011 DOI: 10.1302/0301-620X.98B8.37359.

5. Argaw NA, Shumbash KZ, Asfaw AA, Hawaze Segewkal. Assessment of surgical antimicrobial prophylaxis in Orthopedics and Traumatology Surgical Unit of a Tertiary Care Teaching Hospital in Addis Ababa. BMC Res Notes. 2017; 10:160. Available from: https://www.ncbi.nlm.nih.gov/pubmed/28427474 DOI: 10.1186/s13104-017-2475-2.

6. Das SK, Kurra V, Guttu P, Md. Quadri A, Chowdary NT, Valya B et al. Antibiotic and Analgesic utilization review in an orthopedics in-patient department of a tertiary care teaching hospital in Hyderabad. IJP. Int J Pharm 2016; 6(3):71-9.

7. Leekha S, Terrell CL, Edson RS. General principles of antimicrobial therapy. Mayo clin proc. 2011; 86(2):156-67. Available from: https:// www.ncbi.nlm.nih.gov/pmc/articles/PMC3031442/ Doi:10.4065/mcp.2010.0639.

8. Rajarathna K, Vishwanath M, Ramaswamy A, Kamath SD, Seshu S, Hosthota A et al. Evaluation of WHO Prescribing Indicators Among Orthopaedic Inpatients at a Tertiary Care Hospital. J Chem Pharm Res. 2014; 6(8):278-80. Available from: http://www.jocpr.com/abstract/evaluation-of-whoprescribing-indicators-among-orthopaedic-inpatients-ata-tertiary-care-hospital-3653.html

9. Reji S, Senan A, Philip PT, Kumar A. Utilization of antibiotics in various clinical department in a tertiary teaching rural hospital, Tamil Nadu. WJPPS. 2015; 4(10):1638-48.

10. Ubedulla S, Sekhar NS, Jayasree T, Shankar J, Rohit K. Prescription trends in Department of Orthopedics at tertiary care teaching hospital. JCPR. 2013; 5(11):512-17. Available from:

http://www.jocpr.com/articles/prescription-trends-indepartment-of-orthopedics-at-tertiary-care-teachinghospital.pdf

11. Foote J, Panchoo K, Blair P, Bannister G. Length of Stay Following Primary Total Hip Replacement. Ann R Coll Surg Eng. 2009; 91(6):500-4. Availablefrom: https://www.ncbi.nlm.nih.gov/pmc/articles/PMC2966203 / DOI: 10.1308/003588409X432356.

12. Zhenjun Yali S, Nenpine W, Hao Z. The Rationality and Occasion of Antibiotics Preventive Application in Orthopedics Operations. Chinese Journal of Antibiotics. 2002; 03:1001-8689. 